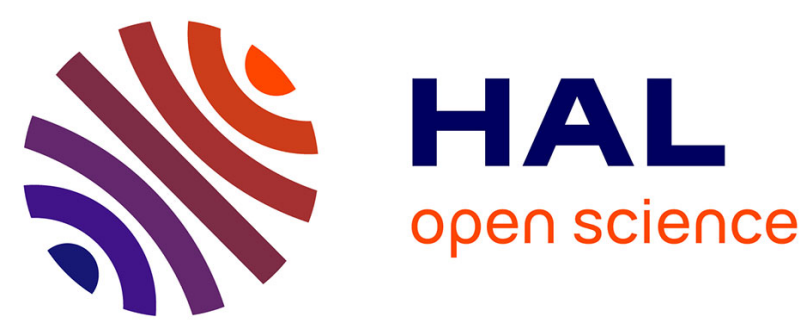

\title{
EFFECT OF FRICTION ON THE STRUCTURAL DYNAMICS OF BUILT-UP STRUCTURES: AN EXPERIMENTAL STUDY
}

\author{
Alfredo Fantetti, Christoph Schwingshackl
}

\section{- To cite this version:}

Alfredo Fantetti, Christoph Schwingshackl. EFFECT OF FRICTION ON THE STRUCTURAL DYNAMICS OF BUILT-UP STRUCTURES: AN EXPERIMENTAL STUDY. ASME Turbo Expo 2020: Turbomachinery Technical Conference and Exposition, Sep 2020, Virtual, United Kingdom. 10.1115/GT2020-14945 . hal-03211077

\section{HAL Id: hal-03211077 \\ https://hal.science/hal-03211077}

Submitted on 28 Apr 2021

HAL is a multi-disciplinary open access archive for the deposit and dissemination of scientific research documents, whether they are published or not. The documents may come from teaching and research institutions in France or abroad, or from public or private research centers.
L'archive ouverte pluridisciplinaire HAL, est destinée au dépôt et à la diffusion de documents scientifiques de niveau recherche, publiés ou non, émanant des établissements d'enseignement et de recherche français ou étrangers, des laboratoires publics ou privés. 
September 21-25, 2020, Virtual, Online

GT2020-14945

\title{
EFFECT OF FRICTION ON THE STRUCTURAL DYNAMICS OF BUILT-UP STRUCTURES: AN EXPERIMENTAL STUDY
}

\author{
Alfredo Fantetti; Christoph Schwingshackl \\ Department of Mechanical Engineering \\ Imperial College London \\ London, United Kindom, SW7 2AZ \\ Email: a.fantetti@imperial.ac.uk
}

\begin{abstract}
Frictional contacts are a major source of uncertainty in the correct prediction of the dynamic response of built-up structures. This uncertainty is partially due to a limited understanding of the effects of friction on dynamic responses. Vice versa, dynamic responses can also affect the frictional behaviour of the interfaces in contact.

In the present study, the mutual relationships between frictional behaviour and structural dynamics are investigated by means of a high frequency friction rig. The rig is characterised by a simple and localised frictional contact that is needed to accurately measure hysteresis loops. Of course, the rig also has its own dynamic response, and consequently represents an excellent test case to gain a better understanding of the correlation between hysteresis loop shapes and their effect upon the dynamics.

Impact hammer tests and shaker tests were performed on the friction rig, and lead to changes in the damping and stiffness of its dynamic response, which were linked to variations in the frictional behaviour of the contact. Furthermore, there was some indication as to how certain resonances of the system might strongly affect the frictional behaviour. In particular, it was $o b-$ served that full sliding causes excitation of structural modes that in turn lead to distortions in the measured hysteresis loops.

These findings confirm the strong relationship between friction and dynamics, thus highlighting the necessity to include a detailed frictional description of contacting interfaces for more accurate modelling of the dynamics of built-up structures.
\end{abstract}

\section{INTRODUCTION}

Friction is a major source of uncertainty for the prediction of the global dynamic response of built-up structures such as turbomachinery [1-3]. Among the many frictional contacts in a turbomachinery, the most relevant are those used in turbine blades for damping purposes such as contacts due to friction dampers [4-8], blade roots $[2,9,10]$ and shrouds $[11,12]$. These contacts have an heavy impact on the dynamic response in terms of shifts in frequency and changes in vibration amplitude [13-15]. Such changes might induce unwanted stresses in the structure, which could eventually lead to high cycle fatigue failures of components $[2,3]$. Therefore, predicting the local frictional response is an essential step to get more accurate dynamic simulations of forced responses.

Most of the current modelling techniques replicate the frictional behaviour by means of the friction hysteresis loop [16], which is the load-deflection curve obtained when plotting the friction force versus the relative tangential displacement occurring between the contacting interfaces. Hysteresis loops are needed to characterize the different frictional regimes, e.g. stick, microslip, gross slip and separation, as these strongly impact the dynamic response.

Unfortunately, contact models need several input parameters to correctly represent the frictional regimes. Friction rigs are still required to measure most of these contact parameters [17-20], because current friction models are not predictive and need to be tuned against experiments [14,21-23], thus leading to designs less accurate and more expensive in terms of time and

*Address all correspondence to this author. 
cost. In addition, a significant amount of uncertainty is also introduced by the large number of nonlinear sources [24, 25], mistuning [4, 26, 27], wear [13], manufacturing tolerances, mounting [10], etc.

For all these reasons, the impact of friction joints on the dynamics becomes quite complicated to predict. It would then be helpful to have a simple structure with only one localised friction joint, so that the effect of friction alone can be observed and thus the hysteretic behaviour can be easily linked to the dynamic response, as already performed in [13] in the case of fretting wear. In this paper, the high frequency friction rig [28] built in the Dynamics Group of Imperial College London is analysed to this purpose. The rig is a relatively simple structure with only one localised friction joint. The simple joint allows to easily get insights on the mutual correlations between hysteresis loops and structural dynamics, with the final goal to obtain more accurate and reliable modelling techniques.

First, the rig setup is shown. Then, the effects of friction on the dynamic response of the system are presented, confirming the existing knowledge and providing some insights for an effective on-site system characterization. Finally, the effects of the system dynamic response on the frictional behaviour are shown, revealing the physical origin of some of the features of the friction hysteresis loops before unknown.

\section{EXPERIMENTAL SETUP}

The high frequency friction rig [28] built in the Dynamics Group of Imperial College London is here used to investigate the correlations between hysteresis loop and dynamic response.

The rig has been built to measure friction input parameters used in nonlinear dynamic analyses for aeroengine and turbomachinery applications. It is characterised by a localised frictional contact needed to accurately measure hysteresis loops. Hysteresis loop are the typical load-deflection curves obtained when plotting the friction force versus the relative tangential displacement, as that in Fig. 1. It is known that hysteresis loops strongly affect the dynamic response of structures [1], and here the dynamic response of the friction rig is studied in detail to experimentally quantify the effects of different hysteresis loop shapes.

\section{Description of the Friction Rig}

The friction rig generates an unidirectional oscillating sliding motion between two cylindrical specimens with a flat-on-flat square contact as those shown in Fig. 2A. A photo of the friction rig is shown in Fig. 2B. The rig is composed of a moving block and a static block. The moving block consists of a moving mass connected to a moving arm by a knife edge, which is needed to avoid any bending resulting from the application of the normal load. The moving mass is excited by means of a shaker, which generates a harmonic excitation. This mass is connected to the

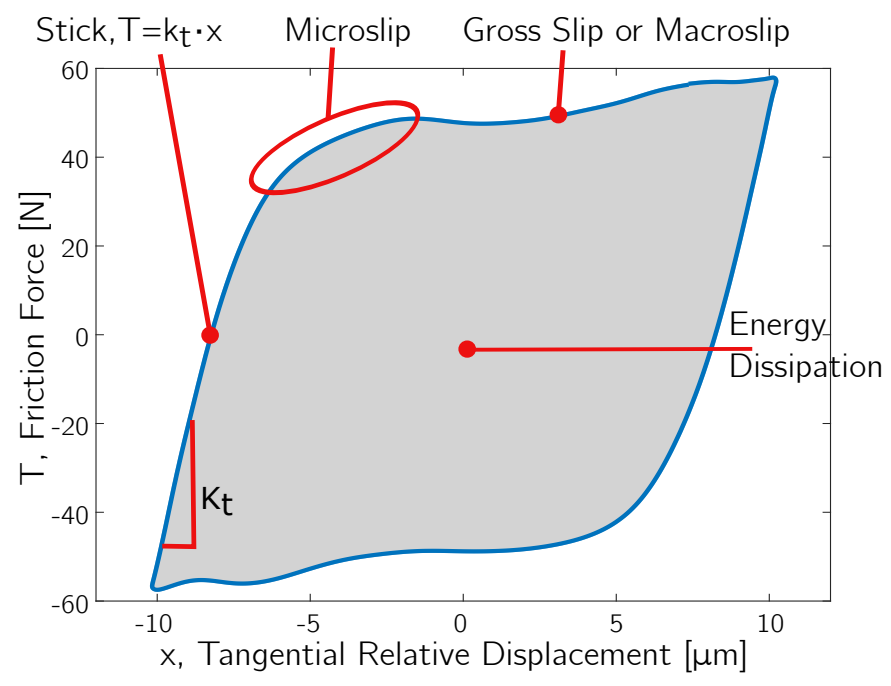

FIGURE 1. Typical hysteresis loop measured from the friction rig [13].

ground by means of two leaf springs that are very flexible, thus allowing large horizontal displacements. A top moving specimen is clamped to the moving arm and slides against a bottom static specimen which is clamped to the static arm.

The relative displacement between the specimens is measured by means of two Laser Doppler Vibrometers (LDVs) that measure velocities, which are integrated to obtain displacements. Laser points are focused very close to the contact interfaces (less than $1 \mathrm{~mm}$ away) by means of mirror laser guides, as shown in Fig. 2A. Such a close position to the contact provides accurate displacement measurements without large influences from the bulk material deformation.

The friction force transmitted through the contact is measured with three load cells that clamp the static arm to the ground. A continuous contact is ensured by applying a normal load with a pneumatic actuator. The load is transmitted by means of push rods that push specimens against each others.

The rig has been designed to minimise the effect of all the joints except the localised frictional contact at the specimen interfaces. A well controlled sliding motion is achievable by means of a displacement control system that varies the excitation force of the shaker. Usually experiments are performed under a harmonic excitation of $100 \mathrm{~Hz}$, as this frequency leads to high quality hysteresis loops. Normal loads can be applied up to $500 \mathrm{~N}$ and a maximum relative sliding distance of $150 \mu \mathrm{m}$ can be achieved. Specimens can reach flat-on-flat nominal areas of contact up to $25 \mathrm{~mm}^{2}$. The rig is able to generate high quality hysteresis loops, which are the essential tools to correctly identify the frictional behaviour of contact interfaces [16], as is well described in the next section. 
A)

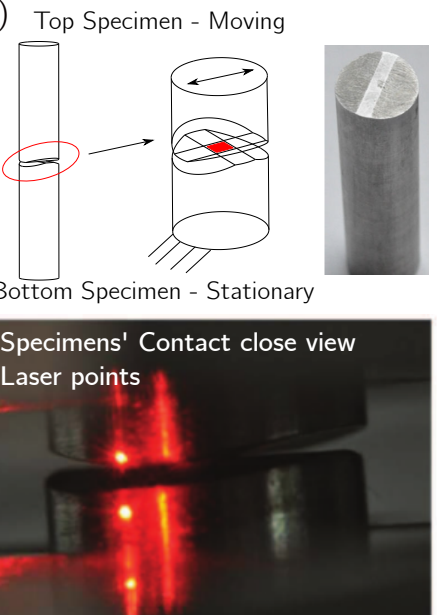

B)

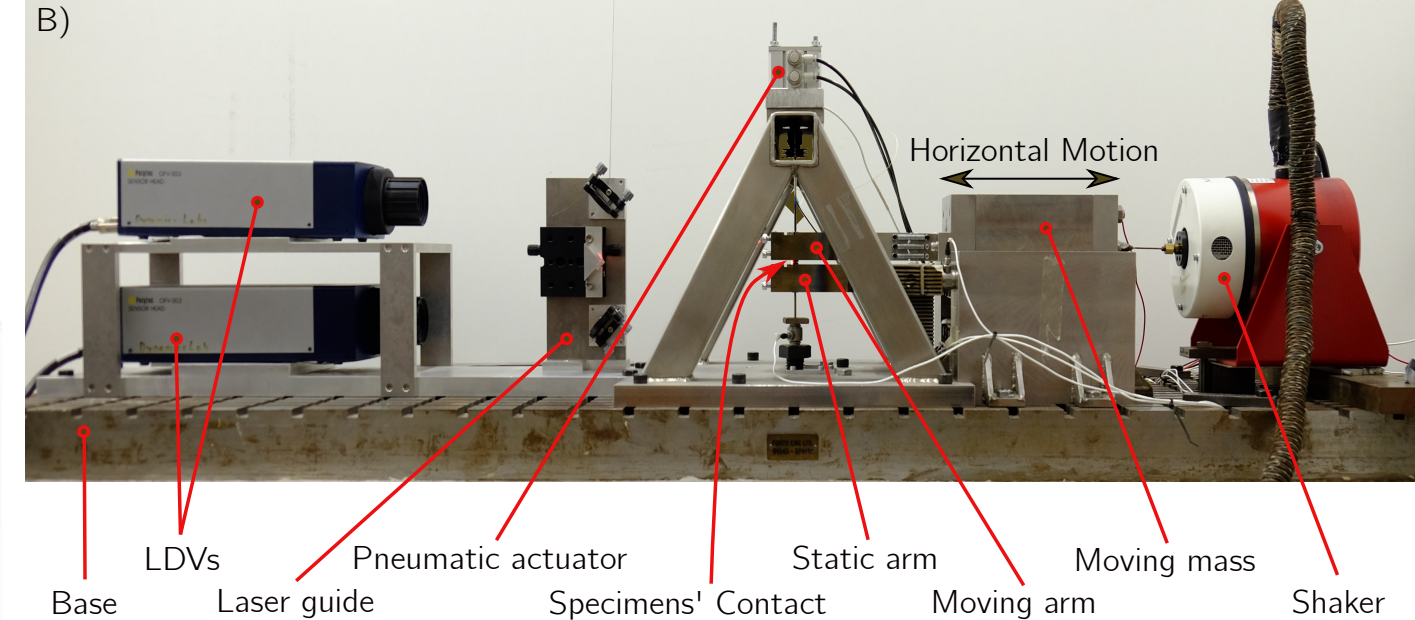

FIGURE 2. A) Specimen interfaces [29]; B) Photo of the friction rig [17].

\section{Description of Frictional Behaviour}

The friction rig generates an oscillating sliding motion that results in hysteresis loops at the specimens' contact interfaces. Here, the physics of the hysteresis loop is briefly described to later understand how the different loops lead to different dynamic responses. A typical hysteresis loop measured from the friction rig is shown in Fig. 1. The loop shape depends on the contribution of the following frictional regimes:

- Stick regime occurs right after the reversal in the sliding direction. In this regime, the friction force is linearly related to the relative displacement by the tangential contact stiffness $k_{t}$. The tangential contact stiffness is evaluated from the slope of this linear behaviour and is due to the elastic deformation of the micro-asperities at the contact interfaces [30] and to the bulk elastic deformation at the macroscopic contact scale [31]. The contact stiffness is an important parameter to predict since it might strongly affect the resonant frequencies of the system [14].

- Microslip occurs during the transition between the stick regime and the gross slip regime, and is due to a partial slip at the contact interfaces. It appears with a progressive flattening of the friction force, which becomes constant during full sliding [1].

- Gross slip occurs when the whole contact experiences a full relative sliding motion. A constant friction force is transmitted and is equal to $\mu N$, where $\mu$ is the friction coefficient and $N$ is the normal load. Due to the friction force, the hysteresis loop dissipates energy, which can be quantified by the area inside the loop itself. This dissipated energy is an important parameter to predict since it affects the damping of the system and the wear of components.

- Separation occurs when the normal load becomes zero and contact interfaces detach. In this case, no friction force is transmitted, but energy could still be dissipated due to impacts. The hysteresis loop will consequently show a $0 \mathrm{~N}$ friction force because the interfaces are not in contact.

It is important to be able to accurately predict the frictional regimes of the hysteresis loop, as they strongly affect the dynamic responses. For example, the energy dissipated affects the damping, while the width of the hysteresis loop (i.e. the sliding amplitude) affects the system stiffness. Those effects are discussed in the following sections.

\section{Description of Rig Dynamic Behaviour}

Before showing the correlations between hysteresis measurements and dynamics, it is important to show the dynamic behaviour of the friction rig. The rig dynamics has been preliminarily modelled by means of a simple 2 degrees of freedom (DOFs) lumped parameter model, which replicates the two main sliding modes of the friction rig. This model is a simple tool needed to facilitate the understanding of the effects of friction on the dynamics and has been first used in [13], where the effect of friction and fretting wear have been numerically simulated and experimentally validated.

Here, this lumped model is validated by a series of hammer tests, which were chosen because they are fast, easy to conduct and can capture instantaneously a large number of modes.

The hammer campaign is shown Fig. 3. The test rig has been dismantled to measure it piece by piece for an easier parameter identification. Hammer impacts at $100 \mathrm{~N}$ were performed on the back of the moving mass. The response amplitude was recorded with accelerometers. A frequency resolution of $0.2 \mathrm{~Hz}$ was used for the data acquisition and for the calculation of the Frequency Response Functions (FRFs). 


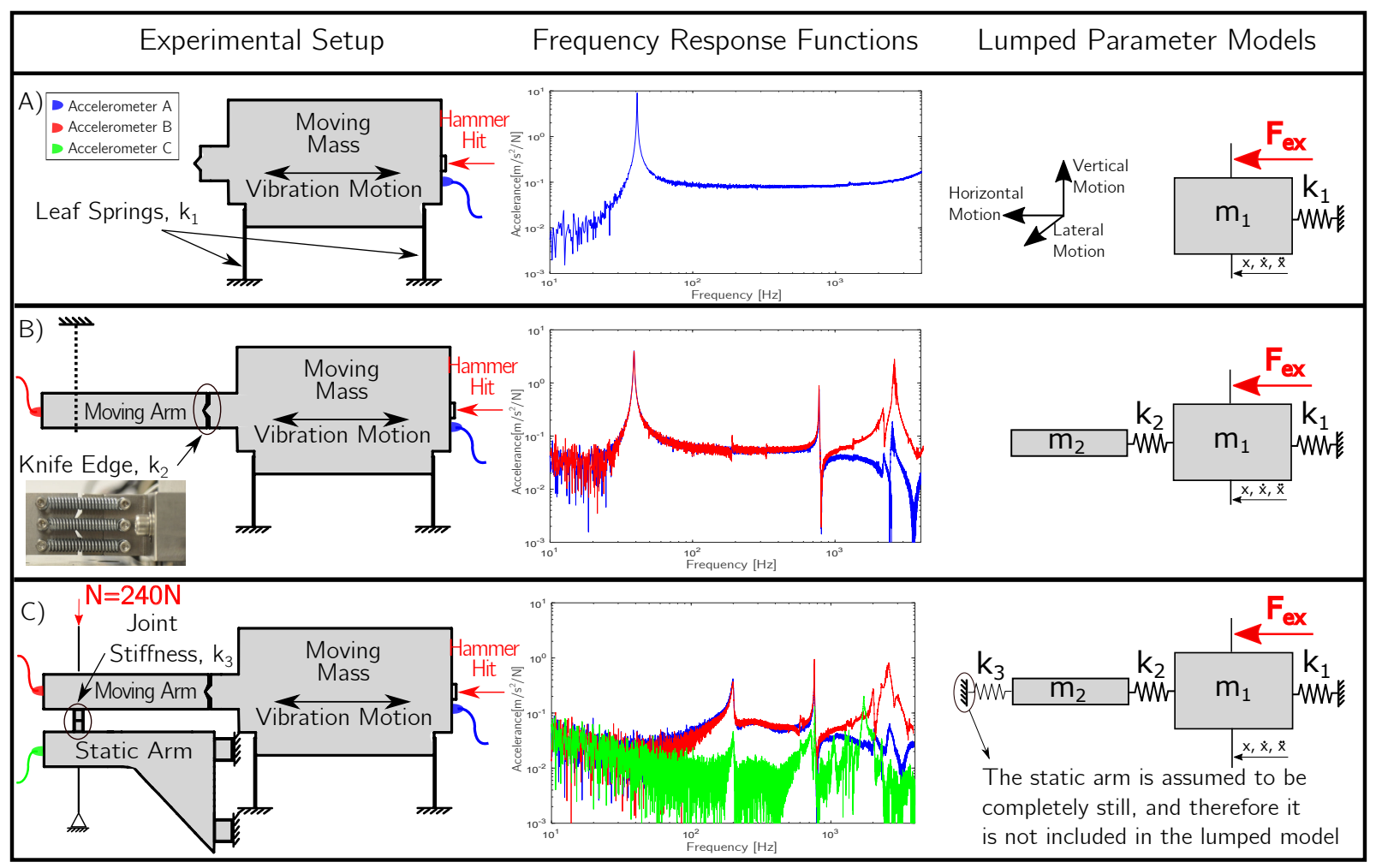

FIGURE 3. Hammer test campaign to evaluate the rig dynamic behaviour: A) moving mass alone; B) moving arm freely suspended on one end and attached to the moving mass on the other end; C) assembly with a very large normal load, which guaranteed a full stuck configuration.

The campaign consisted of the following phases:

Fig. 3A) The FRF of the moving mass solely with the leaf springs was experimentally obtained. Only a single mode appeared below $3000 \mathrm{~Hz}$, suggesting that the moving mass could be modelled as a 1 DOF system with a mass, $m_{1}$, due to the moving mass weight, and a stiffness, $k_{1}$, due to the deflection of the leaf springs. The leaf springs were in fact designed to allow large horizontal motions. The leaf spring stiffness was calculated from the experimental resonant frequency, $f_{1}$, and the known mass of the moving mass using the following formula: $k_{1}=m_{1} \cdot\left(2 \pi f_{1}\right)^{2}$. The damping ratio of this mode was obtained with the half-power bandwidth method [32] from the experimental FRF. Table 1 shows a summary of the lumped parameters obtained from the hammer campaign, and Fig. 3A shows experimental setup, FRF and lumped model.

Fig. 3B) The moving arm was then attached to the mass by means of a knife edge at one end, and on the other end was freely suspended via a fishing wire. A new impact test was performed, and three modes appeared in this new configuration, with two of them representing the in-phase $(40 \mathrm{~Hz})$ and out-of-phase $(2500 \mathrm{~Hz})$ horizontal motion of moving mass and moving arm. The mode at $700 \mathrm{~Hz}$ is an in-plane lateral rotation of the moving arm (see Fig. 3A for the nomenclature on the motion's convention).

The knife edge stiffness, $k_{2}$, was obtained by fitting a 2DOF model to the modes at $40 \mathrm{~Hz}$ and $2500 \mathrm{~Hz}$ from the experimental FRF (keeping unchanged the known values $m_{1}, m_{2}$ and $k_{1}$ ). The damping ratio for this mode was obtained again with the half-power bandwidth method [32].

It is to be noted that the out-of-phase mode at $2500 \mathrm{~Hz}$ is split into two peaks (one at $2000 \mathrm{~Hz}$ ) due to a weak nonlinearity induced by a bolted joint that connects two pieces of the moving mass. This nonlinearity is small compared to the nonlinearity induced by the frictional contact and for this reason is not accounted for in the $2 \mathrm{DOF}$ model, as shown in the next sections.

Fig. 3C) The moving arm was then laid on the static arm and a large normal load of $240 \mathrm{~N}$ was applied to ensure that specimens would stay stuck together during the hammer impact at 100 N. No new modes appeared below $3000 \mathrm{~Hz}$, and therefore the rig could still be modelled as a $2 \mathrm{DOF}$ system. The first mode however shifted from $40 \mathrm{~Hz}$ to $200 \mathrm{~Hz}$. This increase in the resonant frequency was due to a stiffening of 
TABLE 1. 2DOF lumped model of the friction rig.

\begin{tabular}{c|c|c} 
Moving mass & $m_{1}$ & $21.2 \mathrm{~kg}$ \\
\hline Moving arm & $m_{2}$ & $1.2 \mathrm{~kg}$ \\
\hline Leaf spring stiffness & $k_{1}$ & $1.4 \mathrm{~N} / \mu \mathrm{m}$ \\
\hline Knife edge stiffness & $k_{2}$ & $273 \mathrm{~N} / \mu \mathrm{m}$ \\
\hline Damping ratio of $1^{s t}$ mode & $\eta_{1}$ & $0.6 \%$ \\
\hline Damping ratio of $2^{\text {nd }}$ mode & $\eta_{2}$ & $1.5 \%$
\end{tabular}

the system because of the connection to the static arm. In fact, the normal load stiffened the system due to the locking of the contact interfaces (i.e. hysteresis loops were completely stuck).

This stiffening was modelled by adding a stiffness $k_{3}$ on the moving arm. This stiffness is the overall stiffness of the joint and heavily depends on the shape of the hysteresis loop. Since the focus of this investigation is on joint stiffness effects rather than accurate amplitude prediction, the damping due to friction has been neglected in the model at this stage. However, the effect of damping has already been described in detail in [13], where a contact model able to directly replicate hysteresis loops has been successfully used in conjunction with the 2DOF model, instead of the here presented $k_{3}$ approach.

Finally, it should be noticed that the static arm is considered as the ground in the lumped model, since its response (green curve) is more than one order of magnitude lower compared to the moving arm, and therefore can be neglected in the lumped model.

This section shows a description of the friction rig, with a particular interest on its lumped model and dynamic behaviour. A good description of the dynamic behaviour is in fact needed to better understand the effects of friction. The next section shows that the dynamic response is strictly related to the type of frictional regimes (i.e. stick, microslip or gross slip). In particular, the first mode (40-200 Hz) will be analysed more in detail, because it is expected to be the most affected by the frictional contact, since it is in the sliding direction.

\section{EFFECTS OF HYSTERESIS LOOPS ON THE DYNA- MICS}

The aim of this section is to link the dynamic response of the friction rig to the hysteresis loop variations at the contact interface. For this reason, hysteresis loops were measured for different loading conditions under a $100 \mathrm{~Hz}$ harmonic excitation coming from the shaker. After these hysteresis loop recordings, hammer tests were performed to get information on the rig's dynamic behaviour. The measured hysteresis loops were then associated with changes in the dynamic behaviour of the friction rig.

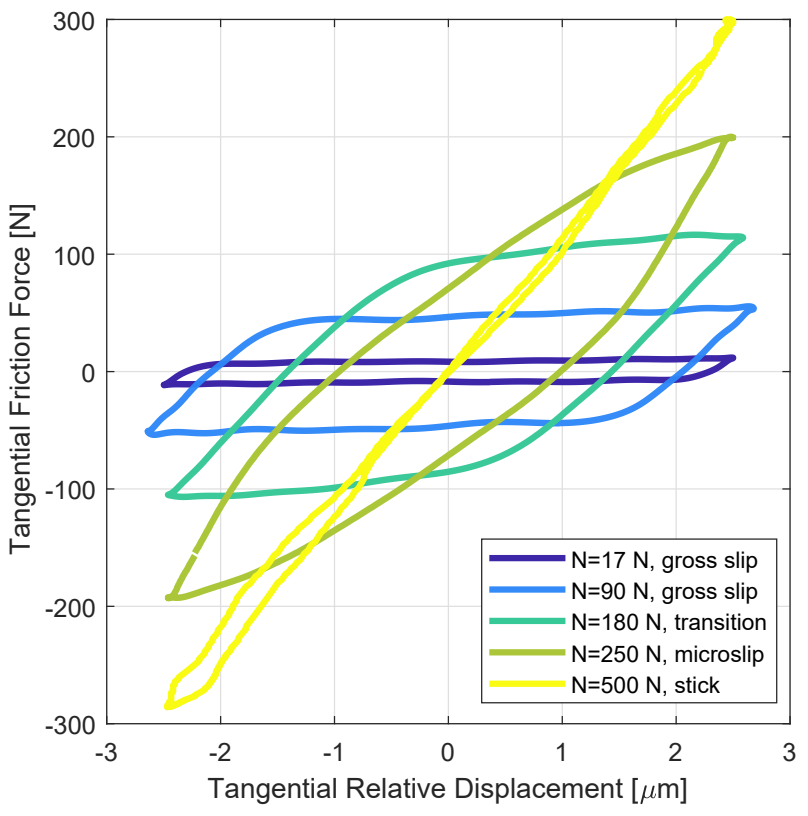

FIGURE 4. Effect of the normal load on hysteresis loops measured at a $100 \mathrm{~Hz}$ harmonic excitation. The transition from stick to microslip to gross slip is visible as the normal load decreases.

\section{Effect of Normal Load}

A first test was conducted to investigate the effect of the normal load on both hysteresis loops and rig dynamics. Hysteresis loops were measured at a $100 \mathrm{~Hz}$ harmonic excitation with a fixed sliding distance of $5 \mu \mathrm{m}$ and varying normal loads. Specimens were made of stainless steel with a flat-of-flat square nominal contact area of $1 \mathrm{~mm}^{2}$.

Figure 4 shows that a transition in the frictional regime appears as the normal load changes. At low normal loads, loops are in the gross slip regime because the sliding limit is small enough to allow full sliding. As the normal load increases, the gross slip reduces until only microslip is present and loops go towards a full stick regime. These changes in the frictional regime strongly affect the dynamic response, and the following dynamic analysis proves it.

Hammer tests were conducted with hammer impacts of $100 \mathrm{~N}$ on the back of the moving mass as shown in the experimental setup of Fig 3C. The response was measured with an accelerometer placed on the moving arm in the sliding direction and different normal loads were investigated. FRFs are shown in Fig. 5. For each normal load, 3 different impacts were performed in order to check the repeatability. Measurements were repeatable in terms of both resonant frequencies and damping, despite the high degree of nonlinearity introduced by the frictional contact. 


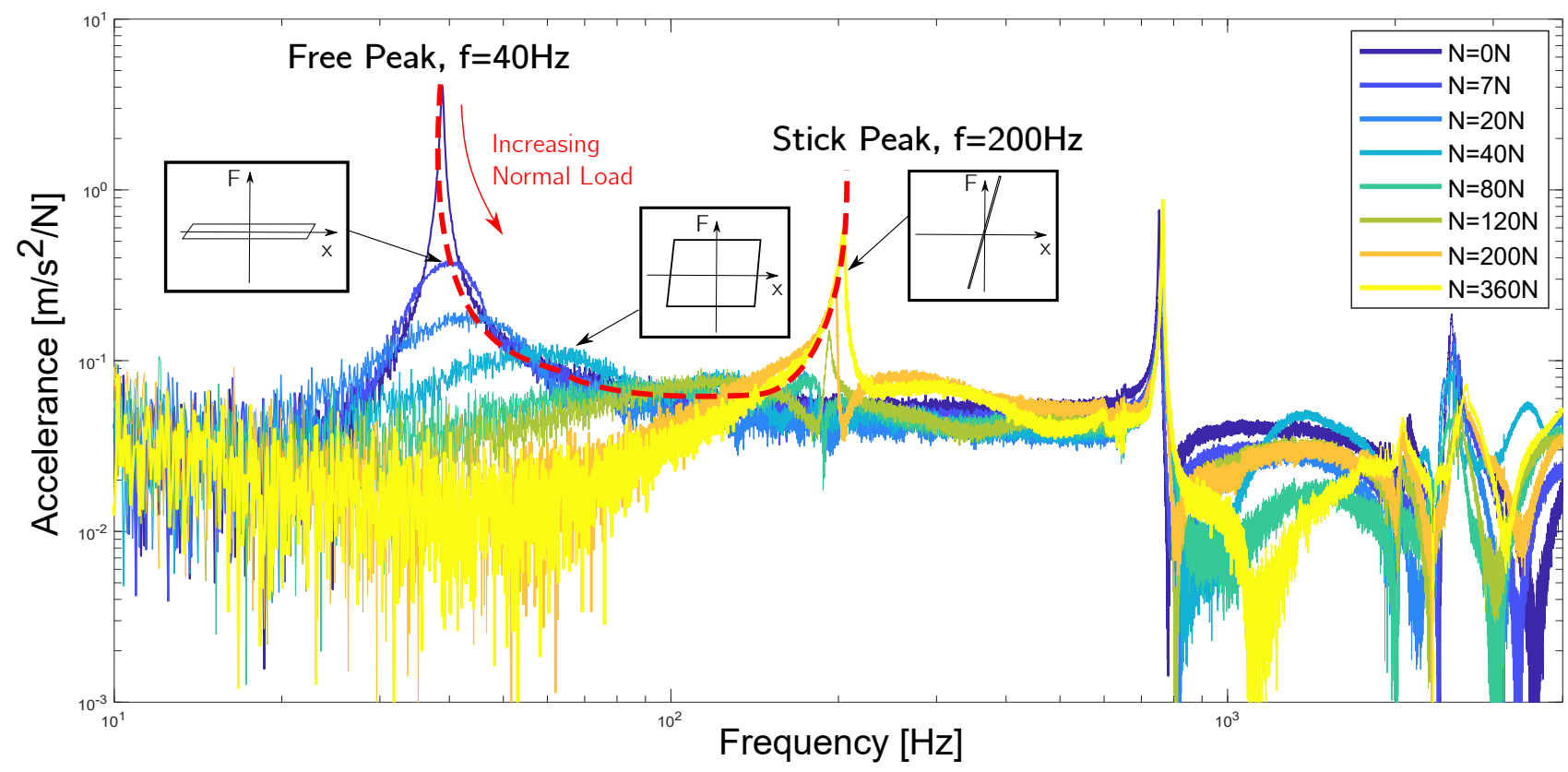

FIGURE 5. FRFs of the friction rig obtained from hammer tests performed at different normal loads and $100 \mathrm{~N}$ of excitation impacts.

It is clear that the first mode shifts from $40 \mathrm{~Hz}$ at very low normal loads to $200 \mathrm{~Hz}$ at very high normal loads. This is due to the different frictional regimes activated by the different normal loads:

- At low normal loads $(N<20 \mathrm{~N})$, the hammer impact $(100 \mathrm{~N})$ is large enough to ensure large sliding. However, the energy dissipated is reduced due to the small friction limit, thus resulting in small damping. The extreme case is achieved for a $0 \mathrm{~N}$ normal load that is the situation of no contact, with no dissipation at all, and hence a "free peak" at $40 \mathrm{~Hz}$.

- At high normal loads ( $>200 \mathrm{~N}$ ), only microslip or full stick is present due to the large friction limit compared to the hammer impact $(100 \mathrm{~N})$. This leads to very small energy dissipated and hence almost no damping that results in a definite "stick peak" at $200 \mathrm{~Hz}$. The resonant frequency increases because the joint is stiffer (as it is not sliding) and the whole system is thus stiffened.

- At medium normal loads (40-120 N), the energy dissipation is maximised thus resulting in very high damping with almost no clear peaks visible in the FRFs.

These results confirm that different frictional regimes strongly affect both resonant frequency and damping of systems with friction joints. In particular:

- Large frequency shifts of more than 5 times were observed due to the frictional regimes transitions. Also damping changes were observed, with resonance amplitudes dropping by more than 10 times due to friction energy dissipation. However, it must be noticed that the investigated structure is a special test case designed purposely to only exhibit nonlinearities due to a localised frictional contact.

- The shifts in frequency and damping are interestingly similar to the behaviour observed in friction dampers applied in turbine blades [8,14,21,23], as both rig and friction dampers exhibit the transition from a "free peak" to a "stick peak".

- The friction rig is used for hysteresis loop measurements usually recorded at a $100 \mathrm{~Hz}$ harmonic excitation. Before the dynamic analysis, it was thought that $100 \mathrm{~Hz}$ was an offresonance value, since the measured hysteresis loops were stable and easily controllable. However, from Fig. 5 it is clear that for some normal load conditions the rig resonance can be at $100 \mathrm{~Hz}$, even though with a highly damped peak that indeed hides the resonance.

These results confirm the existing knowledge on the effects of the normal load on both hysteresis loops and dynamic response. The normal load is one of the main factors affecting friction and dynamics, but it is not the only one. Another important factor is the sliding amplitude, and its effects are discussed in the next section.

\section{Effect of Sliding Amplitude and Excitation Force}

A second test was conducted to investigate the effect of the sliding amplitude on both hysteresis loops and rig dynamics. Blue plots in Fig. 6 are hysteresis loops measured at a $100 \mathrm{~Hz}$ 

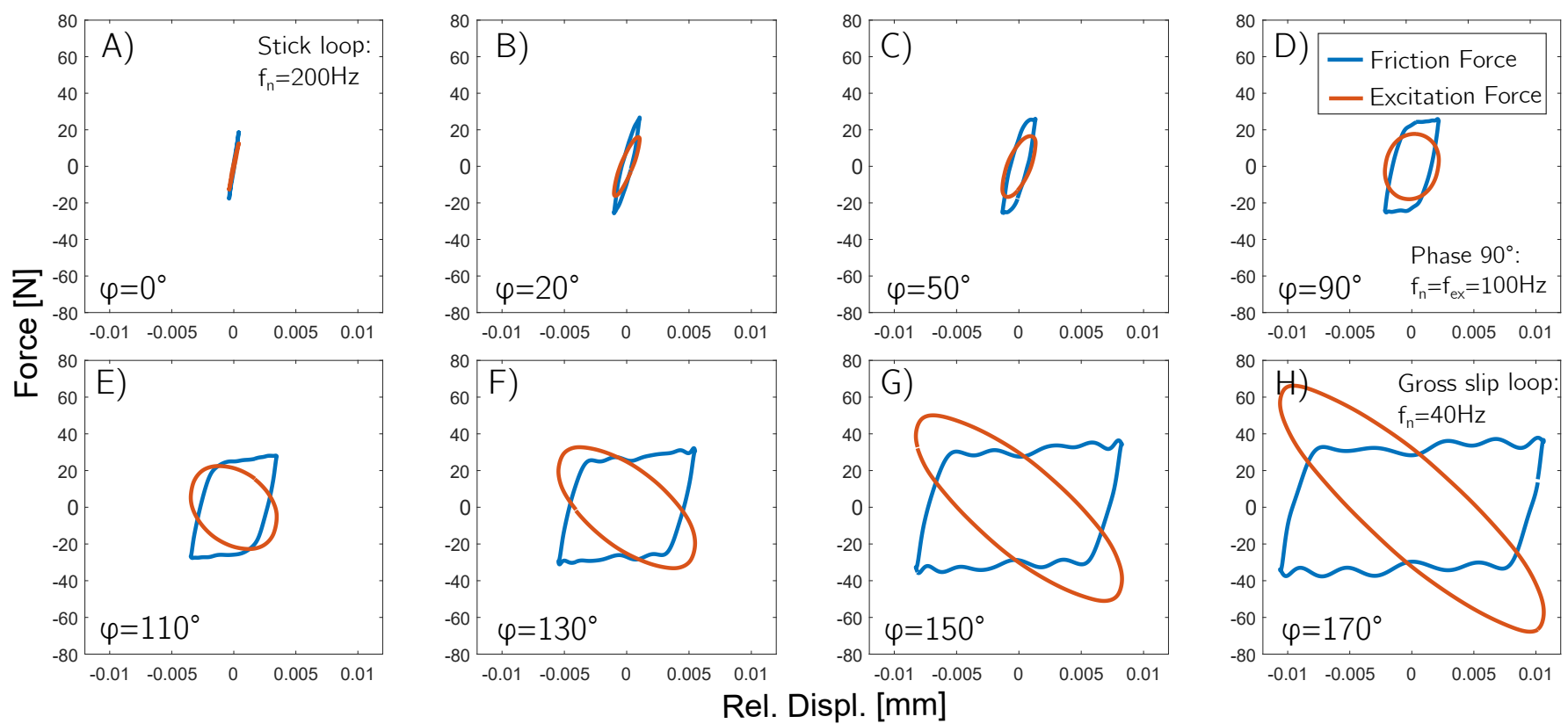

Rel. Displ. [mm]

FIGURE 6. Effect of sliding distance on hysteresis loops (blue plots) and excitation force (orange plots). $\varphi$ is the phase between the excitation force and the displacement. The resonant frequency of the first mode shifts from $200 \mathrm{~Hz}$ to $40 \mathrm{~Hz}$ as the sliding distance increases.

harmonic excitation with a fixed normal load of $40 \mathrm{~N}$ and varying sliding amplitudes. During the hysteresis recordings, also the sinusoidal excitation force from the shaker was recorded and plotted versus the relative sliding distance (orange plots).

The orange plots give insights on the rig dynamics, since they give information on the phase between input force and output displacement. The phase can be visually estimated from the shape of the excitation force-displacement plots. In fact, since both excitation force and displacement are sinusoidal waves, they will generate a line if their phase is zero, a circle when the phase is $90^{\circ}$ and another line (with opposite inclination) when the phase is shifted by $180^{\circ}$. In addition, for every plot in Fig. 6, the exact phase, $\varphi$, is shown, which has been extracted from the comparison between the time signal plots of excitation force and displacement (here not presented).

The trend of the phase of Fig. 6 is explained as follows:

- When the excitation is low, the plot is a line (Fig. 6A) and the phase, $\varphi$, between force and displacement is zero.

- For a medium excitation, the orange plot becomes a circle (Fig. 6D), i.e. the axes of the phase orbit are oriented in the vertical direction. This means that force and displacement are phase shifted by $90^{\circ}$.

- As the excitation increases even more, the friction hysteresis loop enters in the gross slip regime and the displacement gradually goes out of phase with the excitation force until they reach almost a $180^{\circ}$ phase shift (Fig. $6 \mathrm{H}$ ), i.e. the axes of the phase orbit are oriented towards left.
Such shifts between excitation force (input) and displacement (output) indicate that the system response moves through the fixed excitation frequency of $100 \mathrm{~Hz}$. In fact, the resonant frequency changes from $200 \mathrm{~Hz}$ to $40 \mathrm{~Hz}$ while the excitation frequency remains fixed at $100 \mathrm{~Hz}$ and this leads to shifts in the phase. In particular, when the hysteresis loop is in the stick regime, the resonant frequency becomes $200 \mathrm{~Hz}$, as shown in the "stick peak" of Fig. 5, and consequently the excitation force goes in-phase with the displacement. In fact, in this case the excitation frequency of $100 \mathrm{~Hz}$ is below the resonant frequency of $200 \mathrm{~Hz}$ and thus force and displacement are in-phase.

On the contrary, in the case of full sliding, the resonance is at $40 \mathrm{~Hz}$, as shown in the "free peak" of Fig. 5, and consequently the excitation frequency of $100 \mathrm{~Hz}$ is above the resonance, thus leading to a phase shift of $180^{\circ}$ between force end displacement. In other words, the resonant frequency of the friction rig decreases from $200 \mathrm{~Hz}$ to $40 \mathrm{~Hz}$ as the sliding distance increases. The increase in the sliding distance results in wider hysteresis loops that lower the overall stiffness of the joint, and hence lower the resonant frequency of the system.

This experiment confirms that wider hysteresis loops soften the system because the system moves more and thus is less stiff. In order to validate this observation, the average stiffness of each hysteresis loop is used as input in the $k_{3}$ of the 2DOF model of the friction rig, which was shown in Fig. 3C. This average stiffness is obtained by fitting the whole hysteresis loop to a straight line, which represents the overall stiffness of the joint. Of course, 
a more complex contact model that replicates hysteresis loops could have been used instead of the single stiffness $k_{3}$. However, this has already been performed in [13], while here the aim is to show the effects of the joint stiffness alone.

Figure 7 shows the resonant frequencies of the 2DOF model for different $k_{3}$ values. When the loop is stuck (Fig. 6A), the stiffness of $40 \mathrm{~N} / \mu \mathrm{m}$ leads to a resonant frequency quite close to the "stick peak" at $200 \mathrm{~Hz}$. When the loop is in full sliding (Fig. $6 \mathrm{H})$, the low joint average stiffness of $0.5 \mathrm{~N} / \mu \mathrm{m}$ leads to a resonant frequency very close to the "free peak" at $40 \mathrm{~Hz}$. A peak at exactly $40 \mathrm{~Hz}$ would only be reached for a joint stiffness of $0 \mathrm{~N} / \mu \mathrm{m}$ (i.e. a condition of separation with a $0 \mathrm{~N}$ friction force). This good agreement in the resonant frequencies confirms the information obtained from the phase plots, since when the phase is $0^{\circ}$, it means that the excitation frequency $(100 \mathrm{~Hz})$ is below the resonant frequency $(200 \mathrm{~Hz})$, while when the phase is $180^{\circ}$ the excitation frequency $(100 \mathrm{~Hz})$ is above the resonant frequency $(40 \mathrm{~Hz})$.

Finally, the average stiffness of the hysteresis loop of Fig. 6D is also calculated because this loop results in a phase of $90^{\circ}$ and hence it should be the loop for which the excitation frequency equals the resonant frequency. Indeed, the average stiffness of this loop leads to a resonant frequency of $100 \mathrm{~Hz}$ when substituted in $k_{3}$. Hence, this good agreement confirms that phase plots can be used to determine quite accurately the dynamics of the structure via the behaviour at the interface.

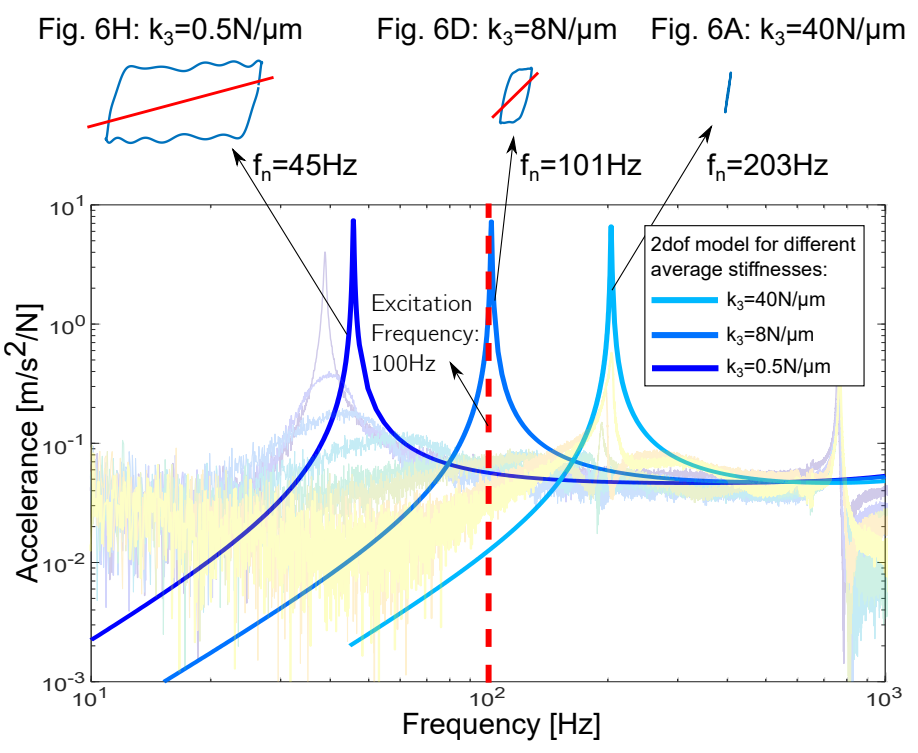

FIGURE 7. FRFs obtained from the linear 2DOF model of the friction rig shown in Fig. 3C. Different values of $k_{3}$ stiffness were obtained by fitting the hysteresis loops to straight lines. In the background, the experimental FRFs of Fig. 5 are shown to see the overlap of the resonant frequencies with the numerical ones.
This investigation has highlighted that:

- The extension of the hysteresis loops directly affects the resonant frequency of the system. Indeed, the hysteresis loop softens or stiffens the system with a stiffness coming from the average slope calculated by fitting the entire loop with a straight line. Changes in this average stiffness explain the shifts in resonant frequencies. In particular, for a larger sliding, the average stiffness is lower and the system softens. On the contrary, for lower sliding amplitudes, the average stiffness increases and the system stiffens. These changes in contact conditions lead to shifts in the resonant frequency.

- The presented "average stiffness approach" is also valid under (dynamically) varying normal loads, which for example can be induced by higher modes contributions. In fact, the average stiffness accounts for all the changes within the hysteresis loops, since it is obtained by fitting the whole hysteresis loop to a straight line, which represents the overall stiffness of the joint. Hence, if there is a significant change in the friction limit, the fitting would take this into account. In future, further tests could be conducted by imposing dynamic varying normal loads to confirm the validity of this approach to more complex loading conditions.

- As previously shown with the static normal load, also the sliding distance strongly affect the resonant frequency and, under certain sliding conditions, the system might be in resonance, although heavily damped. This phenomenon can be advantageously used to get insights on the system dynamics just by knowing the phase shifts between the time signals. For example, phase controls can be implemented to easilier study the dynamics of nonlinear systems [10]. In addition, it is also possible to determine the contact state at the interface quite accurately for a given forcing frequency just by looking at the response phase. However, this is feasible only if it is well known how the joints operates.

These investigations highlight how different frictional regimes strongly affect the dynamics of a structure with a localised friction joint. Unfortunately, the dynamic response can in turn also have an impact on the shape of the hysteresis loops, often leading to distorted measurements. In the next section, the obtained understandings are used in combination with additional measurements to highlight such effects of the dynamics on the shape of the hysteresis loops.

\section{EFFECTS OF THE DYNAMICS ON HYSTERESIS LOOPS}

In this section, the effects of the dynamics on the hysteresis loops are investigated. Previous results have shown massive changes in the dynamic response when different sliding regimes occurred, so vice versa it is expected that the measured loops are being affected by the dynamic response of the system. 

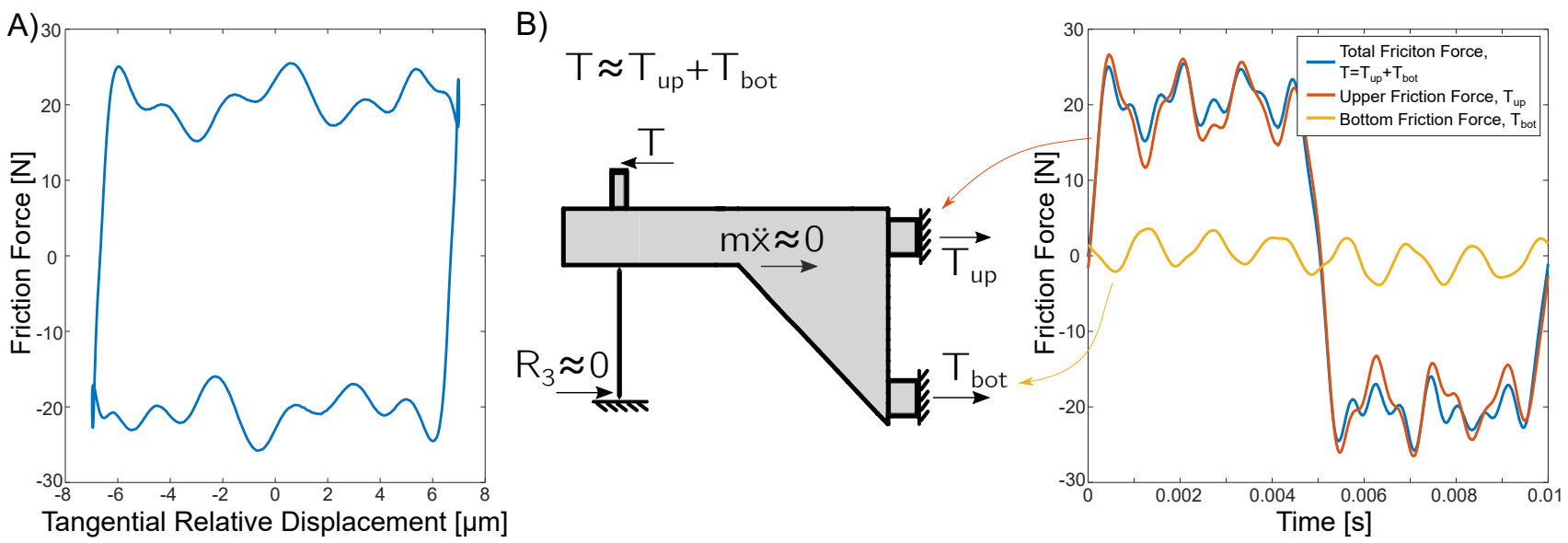

FIGURE 8. A) Hysteresis loop recorded at a $100 \mathrm{~Hz}$ harmonic excitation, $60 \mathrm{~N}$ normal load and $14 \mu \mathrm{m}$ of sliding amplitude. Oscillations in the gross slip region are visible; B) friction force time signal and static arm scheme.

As already described in the previous section, the rig usually operates at $100 \mathrm{~Hz}$, which is the most stable harmonic excitation as it is in the middle of the first resonant frequency transition, where damping is maximum. In fact, even when the resonant frequency is $100 \mathrm{~Hz}$ and coincides with the excitation, the hysteresis loop shape is not affected, because the mode is heavily damped. As a result, the activated nonlinearity can be easily controlled and the extracted contact parameters, i.e. friction coefficient and tangential contact stiffness, will not be affected by this resonance transition.

However, other modes might still affect the hysteresis loop shape. For example, oscillations in the gross slip region of the hysteresis loop are sometimes observed in high frequency sliding tests $[13,33,34]$, as those shown in the hysteresis loop of Fig. $8 \mathrm{~A}$. Here, a possible explanation for the origin of these oscillations is provided: it is hypothesized that such oscillations are not due to the frictional behaviour of the contact interface, but to the dynamic behaviour of the built-up structure.

Looking closer at the friction force data, Fig. 8B shows the time signal of the friction force over the whole loop. The friction force is given by the sum of upper and bottom load cells that measure respectively $T_{u p}$ and $T_{b o t}$ as shown in the schematic of Fig. 8B. When looking at the total friction force, 17 peaks appear over the cycle, thus resulting in a frequency of $1700 \mathrm{~Hz}$ since the excitation frequency is $100 \mathrm{~Hz}$, as confirmed by the fast Fourier transform shown in Fig. 10B. Instead, the upper and bottom force contributions, $T_{u p}$ and $T_{b o t}$, present 7 oscillations over the cycle, thus resulting in a frequency of $700 \mathrm{~Hz}$. Such oscillations are out of phase, indicating a possible bending or rocking mode of the static arm. It must be noticed that several loops were also recorded at larger sliding distances (here not shown) in order to check if these two frequencies changed. The number of peaks remained unchanged, thus indicating that these high frequencies are independent of the sliding distance.

These high frequency oscillations might be due to an excitation of modes close to $700 \mathrm{~Hz}$ and $1700 \mathrm{~Hz}$, especially modes affecting the static arm, which is the only structure between the frictional contact and the load cells that measure the friction force. In fact, if the static arm vibrates, it could affect the force applied on such load cells.

To confirm this hypothesis, Fig. 9 shows the FRF of the static arm obtained from an hammer test at $60 \mathrm{~N}$ of normal load and $100 \mathrm{~N}$ of excitation impact. The impact was performed on the back of the moving mass and the response was measured with accelerometers in the horizontal direction as shown in Fig. 9B. No modes are present up to $600 \mathrm{~Hz}$, thus confirming that shaker experiments conducted at $100 \mathrm{~Hz}$ excitation should not excite any low frequency modes of the static arm. However, the FRF shows two resonant frequencies $(770 \mathrm{~Hz}$ and $1730 \mathrm{~Hz})$, which are close within the $10 \%$ to $700 \mathrm{~Hz}$ and $1700 \mathrm{~Hz}$. These two modes might be the cause of the high frequency oscillations observed in the hysteresis loop. In fact, the friction force activates the odd superharmonics $(700 \mathrm{~Hz}$ and $1700 \mathrm{~Hz})$ of the excitation frequency $(100 \mathrm{~Hz})$, which in turn might excite these two modes. In order to verify this hypothesis, the harmonic excitation was slightly changed from $100 \mathrm{~Hz}$ to $95 \mathrm{~Hz}$. In this second case, the closest odd harmonics to $770 \mathrm{~Hz}$ and $1730 \mathrm{~Hz}$ would be $665 \mathrm{~Hz}$ and $1615 \mathrm{~Hz}$, which are further away compared to the odd harmonics obtained for a $100 \mathrm{~Hz}$ excitation $(700 \mathrm{~Hz}$ and $1700 \mathrm{~Hz})$. Figure 10A shows two hysteresis loops measured at $100 \mathrm{~Hz}$ and $95 \mathrm{~Hz}$. In the second hysteresis loop, it seems that the oscillations at $700 \mathrm{~Hz}$ suddenly disappear, while only the higher frequency oscillations remain. In order to better quantify the change in the frequency content, a fast Fourier transform (fft) of the friction force time signal was performed as shown in Fig. 10B. This $\mathrm{fft}$ is used to quantify the frequency contributions. 

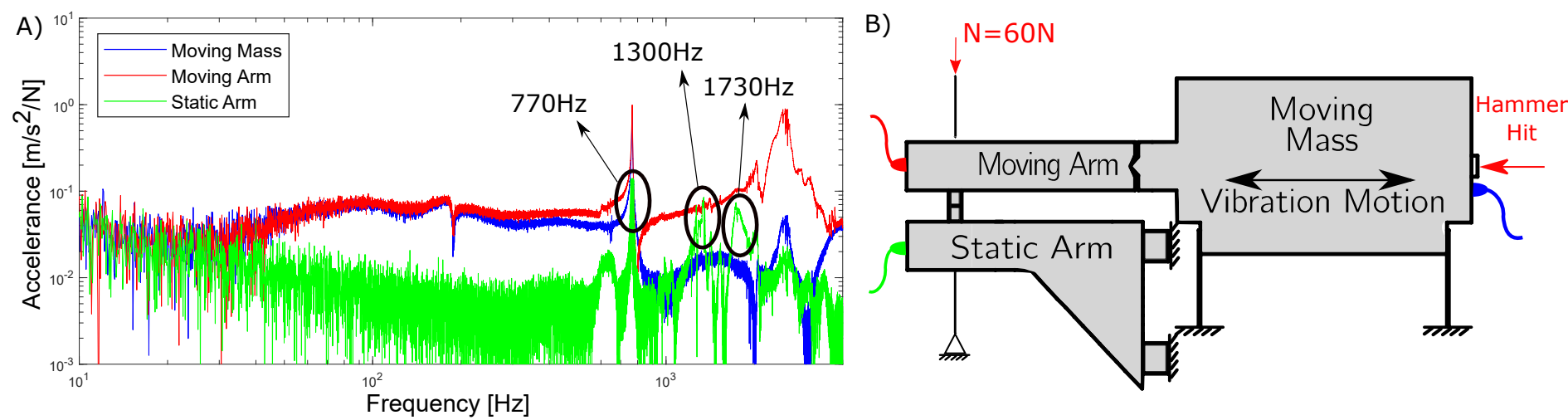

FIGURE 9. A) FRF of the static arm from an hammer test at $60 \mathrm{~N}$ of normal load and $100 \mathrm{~N}$ of excitation impact; B) experimental setup.

A) $\mathrm{f}_{\mathrm{ex}}=100 \mathrm{~Hz}$, odd multiples: 700,1300 and $1700 \mathrm{~Hz}$ $\mathrm{f}_{\mathrm{ex}}=95 \mathrm{~Hz}$, odd multiples: 665,1235 and $1615 \mathrm{~Hz}$

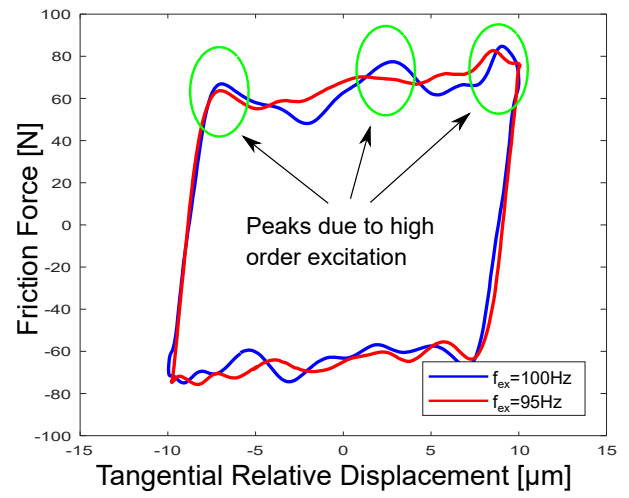

B) Fast Fourier Transform of friction forces, Static arm resonances: 770,1300 and $1733 \mathrm{~Hz}$

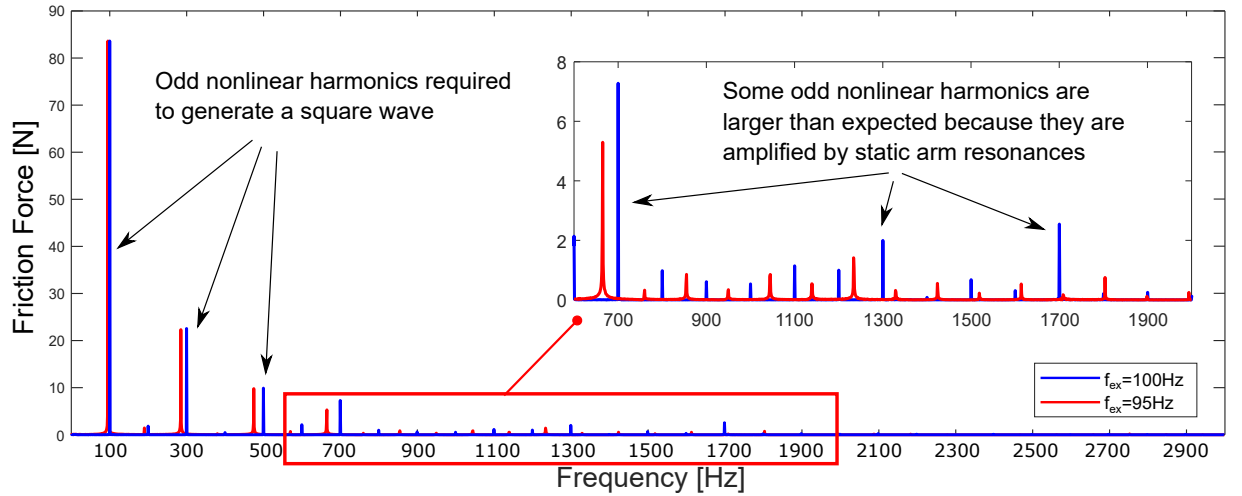

FIGURE 10. A) Hysteresis loops measured at two slightly different excitation frequencies; B) friction forces fft.

There are two sets of frequency contributions:

- The first 3 odd harmonics have a large contribution, which is required to generate a square wave (that is the shape of the friction force in time). In fact, a square wave is obtained by the infinite sum of odd harmonics with decreasing amplitudes [35].

- The subsequent odd harmonic contributions should be almost zero. However, especially for the $100 \mathrm{~Hz}$ case, contributions at $700 \mathrm{~Hz}, 1300 \mathrm{~Hz}$ and $1700 \mathrm{~Hz}$ are larger than they should be. These contributions are probably amplified by a superharmonic resonance with the three modes highlighted in Fig. 9. In addition, it is notable that for the $95 \mathrm{~Hz}$ case, such contributions are much reduced, because the corresponding odd superharmonics are further away from those modes.

This experiment confirms that friction excites the odd superharmonics of the excitation frequency, and if such superharmonics are close to some of the system resonant frequencies, then the system will respond with vibrations that might affect the contact behaviour and the dynamics at the interface. This investigation also suggests that in order to capture such an high frequency behaviour, a large number of harmonics should be used in current modelling approaches for more accurate predictions [14].

It is expected that such high harmonics vibrations do not affect much the extraction of the friction coefficient, because on average they keep the friction limit constant. Unfortunately, they might affect the tangential contact stiffness estimation, as the slope in the stick portion of the hysteresis loop might be sensitive to the presence of a peak or valley due to such vibrations. In other words, the phasing of higher harmonics with respect to the hysteresis loop might affect the extraction of the tangential contact stiffness. In order to minimize such effects in future measurements of hysteresis loops, it is proposed to use excitation levels that do not trigger full sliding, and consequently do not lead to superharmonic excitations. 


\section{CONCLUSIONS}

A high frequency friction rig with a simple localised frictional contact is analysed to provide a better understanding of the mutual relationships between frictional behaviour and dynamic response. It is confirmed that both normal load and relative displacement lead to changes in the frictional regimes (stick, microslip and full sliding), which in turn strongly affect the system's resonant frequency and damping. In particular, changes are observed with up to a five-fold increase in the resonant frequency and up to a ten-fold change in the damping due to different frictional regimes. The dynamic behaviour of the friction rig is the same as that observed in friction dampers, thus confirming the importance of localised friction joints in purposely designed built-up structures.

In addition, even when it is thought that a built-up structure is operating far away from resonance, the excitation frequency may still align with resonance under certain conditions, although not to a visible extent, due to heavy damping. This effect has to be considered for more accurate analyses of built-up structures. Additionally, it is shown that phase plots are a powerful and easy tool to better identify such hidden resonances and to get information related to the frictional regimes.

An easy approach is proposed for modelling shifts in the nonlinear resonances of built-up structures by means of the joint overall stiffness. This overall stiffness can be directly obtained by fitting hysteresis loops to straight lines. Wider loops have lower average stiffnesses, and thus soften the system to a greater extent. As the sliding distance decreases, the average stiffness of the loop increases, therefore stiffening the system. This average stiffness can be used in models as an input parameter to obtain estimations of the nonlinear resonant frequency.

Finally, the origin of the oscillations in the gross slip regime of the hysteresis loops is revealed. It is observed that full sliding causes excitation of structural modes that in turn lead to oscillations in the measured hysteresis loops. Oscillations are induced by the vibration of system components which are excited at their resonant frequencies by the superharmonics generated by the nonlinear friction forces. It is thereby important for designers to consider that the working excitation frequencies (and their odd superharmonics) might excite some resonances of the built-up structure, thus affecting hysteresis loop measurements and consequently the extracted parameters such as the tangential contact stiffness. This effect must be considered for the extraction of more reliable stiffness values and more robusts joint designs.

The findings of this paper experimentally validate the theory of structural dynamics with frictional contacts, confirming the strong relationship between friction and dynamics and thus highlighting the necessity to include a detailed frictional description of contacting interfaces for more accurate modelling of the dynamics of built-up structures.

\section{ACKNOWLEDGMENT}

This project has received funding from the European Union's Horizon 2020 research and innovation programme under the Marie Sklodowska-Curie grant agreement No 721865.

\section{REFERENCES}

[1] Brake, M. R. W., 2018. Introduction to Research on the Mechanics of Jointed Structures. Springer International Publishing, Cham, pp. 3-10.

[2] Srinivasan, A. V., 1997. "Flutter and Resonant Vibration Characteristics of Engine Blades". Journal of Engineering for Gas Turbines and Power, 119(4), p. 742.

[3] Krack, M., Salles, L., and Thouverez, F., 2017. "Vibration Prediction of Bladed Disks Coupled by Friction Joints". Archives of Computational Methods in Engineering, 24(3), pp. 589-636.

[4] Panning, L., Sextro, W., and Popp, K., 2003. "Spatial Dynamics of Tuned and Mistuned Bladed Disks with Cylindrical and Wedge-Shaped Friction Dampers". International Journal of Rotating Machinery, 9(3), pp. 219228.

[5] Gastaldi, C., Fantetti, A., and Berruti, T., 2017. "Forced Response Prediction of Turbine Blades with Flexible Dampers: The Impact of Engineering Modelling Choices". Applied Sciences, 8(1).

[6] Pesaresi, L., Armand, J., Schwingshackl, C. W., Salles, L., and Wong, C., 2018. "An advanced underplatform damper modelling approach based on a microslip contact model". Journal of Sound and Vibration, 436, pp. 327-340.

[7] Botto, D., Gastaldi, C., Gola, M. M., and Umer, M., 2017. "An Experimental Investigation of the Dynamics of a Blade With Two Under-Platform Dampers". Journal of Engineering for Gas Turbines and Power, 140(3), p. 032504.

[8] Fantetti, A., Gastaldi, C., and Berruti, T., 2018. "Modelling and testing flexible friction dampers : challenges and peculiarities". Experimental Techniques, 42(4), pp. 407-419.

[9] Zucca, S., Firrone, C. M., and Gola, M. M., 2012. "Numerical assessment of friction damping at turbine blade root joints by simultaneous calculation of the static and dynamic contact loads". Nonlinear Dynamics, 67(3), feb, pp. 19431955.

[10] Schwarz, S., Kohlmann, L., Hartung, A., Gross, J., Scheel, M., and Krack, M., 2019. "Validation of A Turbine Blade Component Test with Frictional Contacts by PhaseLocked-Loop and Force-Controlled Measurements". Journal of Engineering for Gas Turbines and Power.

[11] Szwedowicz, J., Slowik, S., Mahler, A., and Hulme, C. J., 2005. "Nonlinear Dynamic Analyses of a Gas Turbine Blade for Attainment of Reliable Shroud Coupling”. In Proceedings of GT2005 ASME Turbo Expo 2005: Power for Land, Sea and Air. 
[12] Gross, J., and Krack, M., 2019. "Multi-Wave Vibration Caused by Flutter Instability and Nonlinear Tip Shroud Friction". Journal of Engineering for Gas Turbines and Power.

[13] Fantetti, A., Tamatam, L., Volvert, M., Lawal, I., Liu, L., Salles, L., Brake, M., Schwingshackl, C., and Nowell, D., 2019. "The impact of fretting wear on structural dynamics: Experiment and simulation". Tribology International, 138, pp. 111-124.

[14] Schwingshackl, C. W., Petrov, E. P., and Ewins, D. J., 2012. "Effects of Contact Interface Parameters on Vibration of Turbine Bladed Disks With Underplatform Dampers". Journal of Engineering for Gas Turbines and Power, 134(3), p. 032507.

[15] Zucca, S., Firrone, C. M., and Gola, M. M., 2013. "Modeling underplatform dampers for turbine blades: A refined approach in the frequency domain". Journal of Vibration and Control, 19(7), pp. 1087-1102.

[16] Ewins, D. J., 2018. "A Survey of Contact Hysteresis Measurement Techniques”. In The Mechanics of Jointed Structures, M. R. W. Brake, ed. Springer International Publishing, Cham, pp. 161-192.

[17] Pesaresi, L., Fantetti, A., Cegla, F., Salles, L., and Schwingshackl, C., 2019. "On the Use of Ultrasound Waves to Monitor the Local Dynamics of Friction Joints". Experimental Mechanics.

[18] Lavella, M., Botto, D., and Gola, M. M., 2013. "Design of a high-precision, flat-on-flat fretting test apparatus with high temperature capability". Wear, 302(1-2), pp. 1073-1081.

[19] Kartal, M. E., Mulvihill, D. M., Nowell, D., and Hills, D. A., 2011. "Measurements of pressure and area dependent tangential contact stiffness between rough surfaces using digital image correlation". Tribology International, 44(10), pp. 1188-1198.

[20] Schwingshackl, C. W., Petrov, E. P., and Ewins, D. J., 2010. "Validation of Test Rig Measurements and Prediction Tools for Friction Interface Modelling". Proceedings of ASME TURBOEXPO 2010.

[21] Pesaresi, L., Salles, L., Jones, A., Green, J. S., and Schwingshackl, C. W., 2017. "Modelling the nonlinear behaviour of an underplatform damper test rig for turbine applications". Mechanical Systems and Signal Processing, 85, pp. 662-679.

[22] Szwedowicz, J., Kissel, M., Ravindra, B., and Kellerer, R., 2001. "Estimation of Contact Stiffness and its Role in the Design of a Friction Damper". ASME Turbo Expo, pp. 1-8.

[23] Siewert, C., Panning, L., Schmidt-fellner, A., and Kayser, A., 2006. "The estimation of the contact stiffness for directly and indirectly coupled turbine blading". In Proceedings of ASME Turbo Expo 2006: Power for Land, Sea and Air.

[24] Roncen, T., Sinou, J. J., and Lambelin, J. P., 2018. "Non- linear vibrations of a beam with non-ideal boundary conditions and uncertainties - Modeling, numerical simulations and experiments". Mechanical Systems and Signal Processing, 110, sep, pp. 165-179.

[25] Butlin, T., Spelman, G., Ghaderi, P., Midgley, W. J., and Umehara, R., 2019. "Predicting response bounds for friction-damped gas turbine blades with uncertain friction coupling". Journal of Sound and Vibration, 440, feb, pp. 399-411.

[26] Jareland, M. H., and Csaba, G., 2000. "Friction Damper Mistuning of a Bladed Disk and Optimization with respect to Wear". In Proceedings of ASME Turbo Expo 2000.

[27] Vargiu, P., Firrone, C. M., Zucca, S., and Gola, M. M., 2011. "A reduced order model based on sector mistuning for the dynamic analysis of mistuned bladed disks". International Journal of Mechanical Sciences, 53(8), aug, pp. 639-646.

[28] Schwingshackl, C. W., 2012. "Measurement of friction contact parameters for nonlinear dynamic analysis". Topics in Modal Analysis I, 5, pp. 167-177.

[29] Gimpl, V., 2019. "Substructuring Techniques to Extract Friction Contact Parameters for Aero-Engine Applications". PhD thesis, Technical University of Munich.

[30] Greenwood, J., and Williamson, J., 1966. "Contact of nominally flat surfaces". Proceedings of the Royal Society London A, 1442(295), pp. 300-319.

[31] O’Connor, J. J., and Johnson, K. L., 1963. “The role of surface asperities in transmitting tangential forces between metals". Wear, 6(2), pp. 118-139.

[32] Ewins, D. J., 1984. Modal testing: theory and practice. Vol. 15. Letchworth: Research studies press.

[33] Gastaldi, C., and Gola, M. M., 2017. "A random sampling strategy for tuning contact parameters of underplatform dampers". In Proceedings of ASME Turbo Expo 2015: Turbine Technical Conference and Exposition, pp. 1-12.

[34] Hager, C. H., Sanders, J. H., and Sharma, S., 2004. "Characterization of mixed and gross slip fretting wear regimes in Ti6Al4V interfaces at room temperature". Wear, 257(1-2), pp. 167-180.

[35] Randall, R. B., 1987. Frequency Analysis (Bruel \& Kjaer). Preventative maintenance program handbook (IRD Mechanalysis). 